\title{
Connection Time Estimation between Nodes in VDTN
}

\author{
Adnan $\mathrm{Ali}^{1}$, Muhammad Shakil ${ }^{2}$, Hamaad Rafique ${ }^{3}$, Sehrish Munawar Cheema ${ }^{4}$ \\ Department of Computing and Information Technology, University of Sialkot, Sialkot, Pakistan ${ }^{1,3,4}$ \\ MediaNet Lab, Korea Advanced Institute of Science and Technology, Daejeon, Republic of Korea ${ }^{2}$
}

\begin{abstract}
Vehicular delay tolerant network (VDTN) is a widely used communication standard for the scenarios where no end to end path is available between nodes. Data is sent from one node to another node using routing protocols of VDTN. These routing protocols use different decision metrics. Based on these metrics, it is chosen whether to send data to connected node or find another suitable candidate. These metrices are Time to live (TTL), geographical information, destination utility, relay utility, meeting prediction, total and remaining buffer size and many other. Different routing protocols use a different combination of metrics. In this paper, a metric called "estimation-time" is introduced. The "estimation-time" is assessed at the encounter of two nodes. Nodes may decide based on that whether to send data or not. This metric can be used in routing decisions. The simulations results are above $88 \%$ which proves "estimationtime" metric is calculated correctly.
\end{abstract}

Keywords-Vehicular delay-tolerant network; delay tolerant network; smart transportation

\section{INTRODUCTION}

Smart cities have been establishing in a world very rapidly, more than a thousand cities have been established so for globally [1]. In the world of internet of things (IoT) and smart cities, transportation between them is an essential part of the broader spectrum. IoT is not limited to just cities, it also aims to connect rural areas and places. Vehicular ad-hoc networks play important role in smart transportation for data transfer purposes, as their protocols work best in urban environments where nodes are dense however when it comes to sparse nodes, then a better approach is used named vehicular delay tolerant network (VDTN). Delay tolerant networks (DTN) are the type of ad-hoc networks, where end to end connectivity does not exist. DTN has a message-oriented overlay layer called "Bundle Layer" employs a store, carry and forward message switching paradigm that moves messages from node to node, along with a path that eventually reaches the destination [2]. It works as follows that a source node creates messages which are called bundles and store that message in its internal storage until it meets with another node. On meetup, it forwards data to receiving node, it keeps happening until data delivered to the destination node or its time to live (TTL) expires. In this type of network, nodes are sparse, distances are long, meeting probability of end to end node is very low due to dynamic changing topologies, delays are long and variable, high latency, asymmetric data rate, and high error rates are common. VDTN hold the same properties as the VDTN is when nodes are vehicles in delay tolerant network. In VDTN, vehicles carry the data and deliver it from one place to another place. VDTNs are supposed to perform better in poor conditions because it works with the store, carry and forward paradigm. VDTN register short contact durations and experience rapid changes in the network [3].

Normally vehicles travel at different speeds which may vary based on scenarios.

- Inside the city, speed may be less, as compared to outside the city.

- Traffic density will also have an impact on speed.

- Highway scenario speed will be faster.

- Time of day also affects driving behavior.

Vehicles movements are unpredictable; however, few properties are common for sure that they will travel only on paths or roads with some movement speed along with other vehicles. This speed property can be used. In normal networks, it is not easy to find the connection time of two nodes due to the unpredictable behavior of a user. A user may disconnect or reconnect anytime. Vehicles have some pattern. Like, they travel on roads, with some speed and cross each other randomly depending on their speed and quantity. With this speed factor, estimation-time is calculated. Security of the VANET and VDTN is important. Authors of [4], [5], [6] and [7] discuss about security and [4] proposed a novel biologically-inspired spider monkey time synchronization (SMTS) techniques for largescale VANETs.

At the time of connectivity between any two nodes in VDTN, if communication time can be estimated, that would be handy in routing decisions. The sender node will be able to take a decision that, rather to send data to the receiver node or to find suitable node. Let suppose there is very short time, 1 or 2 seconds and bundle size is bigger 10 or $20 \mathrm{MB}$, so it is not good idea to send the data as the connection will be terminated before data delivery completion. In this type of scenario, it's better to find another suitable candidate rather than wasting the bandwidth on the previous node. A study [8] shows the connection time between vehicles using $802.11 \mathrm{~g}$ and says if vehicles are $20 \mathrm{KM} / \mathrm{h}$ then time is about $40 \mathrm{~s}$ and at $40 \mathrm{KM} / \mathrm{h}$ time is just $15 \mathrm{~s}$, if speed is being increasing then time will keep decreasing and at $60 \mathrm{KM} / \mathrm{h}$ speed time reduced to $11 \mathrm{~s}$. With TCP 4 out of 10 attempts was not useful because TCP is connection-oriented and take more time, however with UDP results were better. So, the authors of [2] give a solution of message fragmentation. This work suggests if "estimatedtime" is calculated correctly, fragmentation will not be 
required any longer and still it will be possible to send data with a good success ratio. In addition, if bundle size can be limited according to the data rate and estimated time, fragmentation may never be required. The experiments show that it is possible to predict the "estimation-time" with good accuracy. This Paper is divided as Sections I, consists of introduction. Section II Literature Review, Section III Methodology, Section IV Results and Discussion, Section V Conclusion, and Future work.

\section{LITERATURE REVIEW}

VDTN is an active in research area for more than a decade, Different authors and research groups are working in this field to make this filed better. The paper [9] is about dropping policies of messages that decides which message should be dropped from the buffer when the buffer is full. Relay nodes [10] are also being used for better performance. Relay nodes are fix nodes who just receive and forward data, these are not terminal nodes. There are multiple parameters being used in different routing protocols for routing decisions. Like DAWN and GeOpps basic forwarding metrics are the density of nodes. TABLE I shows some routing schemes for forwarding metrics.

TABLE I. ROUTING SCHEMES WITH ForWARding METRICS [6]

\begin{tabular}{|l|l|l|}
\hline $\begin{array}{l}\text { Sr. } \\
\text { No }\end{array}$ & Scheme Name & Forwarding Metrics \\
\hline $\mathbf{1}$ & PBRS[11] & Velocity-based Probability \\
\hline $\mathbf{2}$ & ACSF[12] & $\begin{array}{l}\text { Minimum-outage } \\
\text { time of the node }\end{array}$ \\
\hline $\mathbf{3}$ & DARCC [13] & $\begin{array}{l}\text { Location of destination moving direction of } \\
\text { nodes }\end{array}$ \\
\hline $\mathbf{4}$ & DAWN[14] & Density of nodes \\
\hline $\mathbf{5}$ & GeOpps [15] & Density of nodes \\
\hline $\mathbf{6}$ & GeoSpray [16] & Density of nodes and Different Data Size \\
\hline
\end{tabular}

This paper is about introducing a parameter named "Estimated Time". Some routing protocols will be discussed along with their parameters or metrics to highlight the importance of parameters that how it can make a routing protocol top or flop. DTN based routing protocols can be categorized based on the number of copies of the bundle in the network. Single copy and multicopy are two major types of routing protocols. Single copy schemes uphold a single copy of the data bundle in the network, in opposition multi-copy holds multiple copies. First contact [17] and direct delivery [18] are examples of a single copy. These both do not hold any network knowledge for routing decisions. Examples of multicopy are Epidemic [19], Spray and wait [20] and PRoPHET [21].

Epidemic [19] is a routing protocol which does not contain any prior knowledge of the network. Each node just has a list of bundles with it. Whenever it encounters a new node, they both exchange the missing bundles. In the end, every node has every bundle including destination node. This technique is not good because, it is flooding base and it causes excessive bandwidth usage and some nodes will still have the data even the data is sent to destination. Although it is best in terms of delivery rate that it surely delivers the message. That's why this protocol is used as a benchmark to compare other protocols [22]. As per this paper [23], performance of Epidemic is better than other protocols where delays are greater. Spray and wait [20] limits the replication, it consists of two phases, In "spray phase" message copies are generated and sent to L nodes if the destination node is found in this step then fine else "wait" phase starts. It waits until the destination node is found. Another routing protocol which uses past encounters history for delivery predictability is known as PRoPHET [21], in this protocol, which node with higher probability gets the data. MaxProp is another forwarding base algorithm, it works with initial meeting probability which is being set for each node, then this information is shared with neighbors. It is buffer consuming and perform well with larger buffer size [24].

There are some routing protocols which uses global positioning systems GPS along with other parameters. In this class of routing protocols, decisions are taken based on assumption that every step is towards the destination node. GeOpps [15] and Geo-Spray [16] are location-based routing protocols. GeoVDM [25] is a comparatively new protocol which is also GPS base. DAWN [14] is a local capacity constraint density adaptive DTN routing algorithm. It improves the packet delivery ratio within the deadline where packet network capacity is limited. Mobile nodes decide, number of packets to broadcast based on local density information. In this paper [26] the author introduced a new parameter called the trend of delivery, which is used then for routing decisions. Max-Util [27] is a utility based algorithm proposed in this paper whereas their routing decisions are based on parameters like destination utility, relay utility, buffer utility, contact, and overall utility. There is another paper [28] which is related to network management, the network is managed by selecting the managers who are stable nodes. A priority-based scheduling and drop policy are proposed in this paper [29] along with a hybrid routing algorithm that routes messages which are scheduled built on priorities. Performance of routing protocols is being compared in this paper [30] while considering different parameters like time to live, distance (long and short) and a number of hops. Taking the routing decisions while maintaining privacy is considered in this paper [31] routing protocol named ePRIVO. It ensures the link privacy, binary anonymization, and neighborhood randomization, and attribute privacy by means of the Paillier homomorphic encryption scheme. Nodes share their information in the homomorphic encrypted form.

\section{Methodology}

Vehicles may travel at different speeds, depending on the traveling area, time and vehicle's density. In the city, vehicle may travel slower than outside the city, at early morning traveling speed may be different from office timings. Also, vehicle speeds are dependent on traffic density, and depending on driver's behavior speed may vary too [32] [33]. By keeping this in consideration and to make it closer to reality variable speeds are assigned to vehicles ranging from $30 \mathrm{KM} / \mathrm{h}$ to $70 \mathrm{KM} / \mathrm{h}$ in $10 \mathrm{KM} / \mathrm{h}$ chunks. So, if speed is between $30-$ $40 \mathrm{KM} / \mathrm{h}$ then it means the speed of both vehicles could be any value between 30 and $40 \mathrm{KM} / \mathrm{h}$. It is not necessary that both vehicles are traveling at the same speed. 
The speed and connection time are inversely proportional to each other, greater the speed value mean lesser the connection time [33].

Here in this research, main focused is two type of times.

- Connection time

- Estimation time

\section{A. Connection Time}

This is the actual communication time of two vehicle nodes and it can be found in ONE (Opportunistic Network Environment) simulator [34]. This is time between the start of the communication to the end of the communication, and only can be found when connection get terminated. This time can be used for time prediction of future encounters. In past if two nodes meet frequently and communicate with each other for long time, then their future behavior can be foretold. The "estimation time" and connection time are compared to find out accuracy of proposed methodology.

\section{B. Estimation Time}

Estimation time is predicated time, it is estimated by Equation 2, at starting phase of meeting encounter. This time can be used for routing decisions. At the moment of decisionmaking process, if somehow, it is predicted that this communication is going to last for a specific interval like 4 or 5 seconds then the node can decide how much data it should send, or not send at all. Estimated connection time can be assessed using vehicle speed and distance. Vehicles speed and location can be gotten in ONE simulator [34]. From this location distance between two vehicles is found. So, there is speed and distance. Now here is an equation to find the estimated time.

Ts=Total Speed

Ss= Data sending node Speed (Data initiating node)

$\mathrm{Rs}=$ Dara receiving node speed

$\mathrm{Et}=$ Estimated Time

$\mathrm{D}=$ Distance between sender and receiver

$\mathrm{T} \sigma=S s+R s$

$E t=\frac{D}{T s \times 2}$

It is observed in simulations that estimated time changes with vehicle speed. TABLE II shows the estimated time for different speeds.

TABLE II. ESTIMATED TIME FOR DIFFERENT SPEEDS IN SECONDS

\begin{tabular}{|l|l|l|l|l|l|l|l|l|}
\hline $\begin{array}{l}\text { Sr. } \\
\text { No }\end{array}$ & $\begin{array}{l}\text { Vehicle/s } \\
\text { Speed/s }\end{array}$ & \multicolumn{2}{|l|}{ Time with Number of Vehicles } & \multirow{2}{*}{$\begin{array}{l}\text { Average } \\
\text { Time }\end{array}$} \\
\hline \multirow{2}{*}{} & \multicolumn{2}{|l|}{ Vehicles } \\
\cline { 2 - 7 } & 2 & 3 & 4 & 13 & 14 & 15 & \\
\cline { 2 - 7 } & Time \\
\hline 1 & $30-40$ & 8.91 & 9.07 & 9.10 & 9.12 & 9.16 & 9.15 & 9.072 \\
\hline 2 & $41-50$ & 6.75 & 6.86 & 6.69 & 6.74 & 6.75 & 6.75 & 6.758 \\
\hline 3 & $51-60$ & 5.50 & 5.50 & 5.45 & 5.45 & 5.44 & 5.47 & 5.468 \\
\hline 4 & $61-70$ & 4.50 & 4.65 & 4.57 & 4.60 & 4.59 & 4.59 & 4.582 \\
\hline \multicolumn{8}{|l|}{ Average Estimated Time of Sr. No 1 to 4} \\
\hline
\end{tabular}

\section{Vehicle Movement Scenario}

There could be these three scenarios for vehicles traveling on road. Some of them will be moving and some may be stationary as given wait time is between 0 to 120 seconds as mentioned in TABLE IV. So, vehicles will be stationary between that wait time, while moving they will be traveling in different directions. There could be these possible scenarios.

1) Scenario 1: One vehicle is moving and other is stationary. As shown in Fig. 1 that V2 is stationary and V1 is passing by. In this scenario, time will be greater than when both vehicles are moving, as explained in [33] and as per Equation 1, One vehicle speed will becomme zero, while other node will have some value greater than zero, and estimated time will be higher.

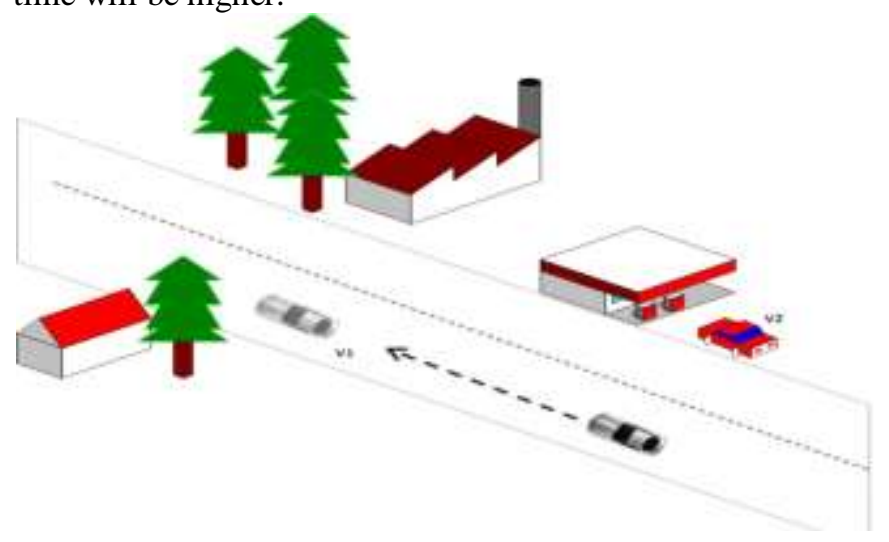

Fig. 1. V1 is Moving and V2 is Stationary.

2) Scenario 2: In this scenario, both vehicles are stationary, after running multiple simulations, there was no such scenario where both vehicles were not moving and having communication. So, this is not considered in this paper.

3) Scenario 3: Both Vehicles are moving and having active communication, this could have further three subscenarios.

a) Both are moving in the same direction.

b) Both are moving in the opposite direction

c) Both are crossing each other at the intersection.

4) Scenario A: It is possible that both vehicles are traveling in the same direction and having communication as shown in Fig. 2. In this scenario, time estimation is harder. Because it is never known which vehicle will take a turn and when. After running simulations, it is observed that in some cases "connection time" values are too much higher than mean value. As per authors observations, the reason for these out of the box time values is, both vehicles are going in the same direction for a comparatively longer time. This does not happen frequently in city base scenarios, as it can be observed from the graph. The good thing is in most of the cases its value will be bigger than the estimated time. TABLE II shows the estimated time's value never goes greater than 10 seconds but in connection time with two vehicles and speed between 60 and $70 \mathrm{KM} / \mathrm{h}$ the connection time is 86 seconds. So, it is very clear that in this case vehicles were traveling in the same 
direction. For this case, assumption for this paper is that actual connection time will be always greater than estimated time.

5) Scenario $B$ and Scenario $C$ : In these two scenarios, time will be limited and calculate-able. Here vehicles will be traveling towards each other and after meeting they will be separated as shown in Fig. 3 and Fig. 4 While vehicles are traveling towards each other, their speeds will be combined.

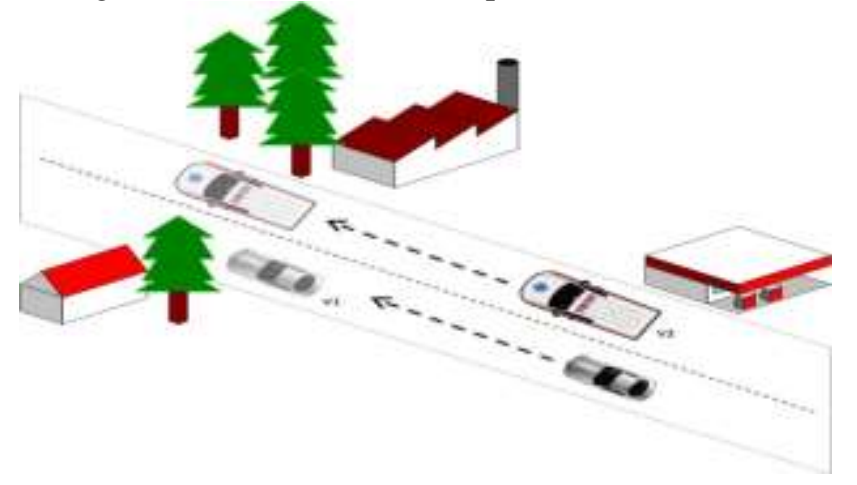

Fig. 2. Both Vehicle are Moving in Same Direction.

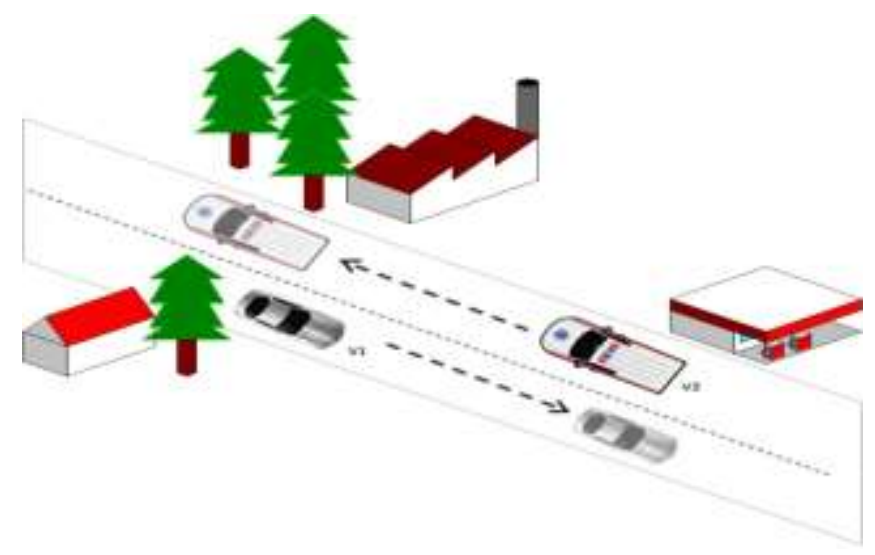

Fig. 3. Both Vehicles Moving in Opposite Direction.

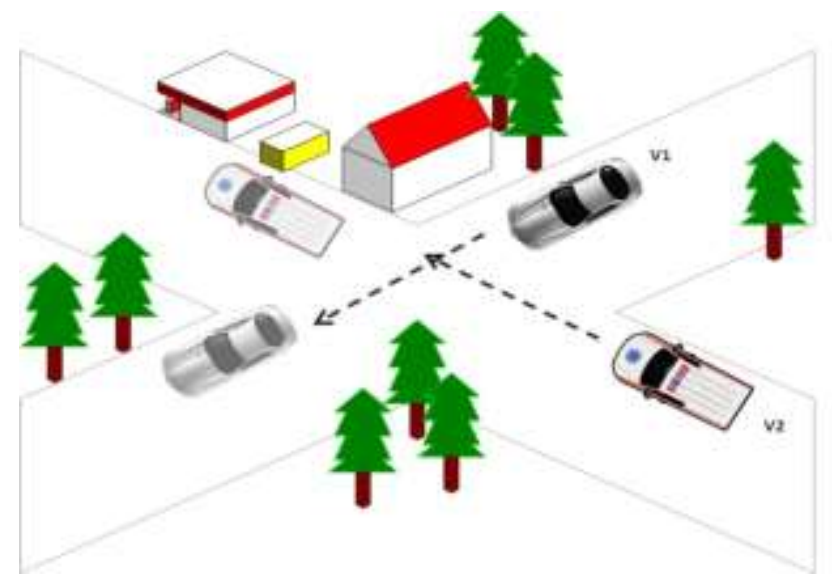

Fig. 4. Both Vehicles are Crossing Each other At Intersection.

\section{RESUlts AND Discussion}

Number of vehicles in scenario and vehicle speed is directly proportional to number of encounters. More vehicles mean more meeting chances and more speed also creates more chances of an encounter. TABLE III shows the number of encounters for different simulation scenarios.

\section{A. Simulations Setup}

Tool used for simulations is Opportunistic Network Environment (ONE) simulator; it is java-based simulator which has a configuration file named "default_setting.txt" where metrices values can be changed. TABLE IV shows values used for these simulations. All other values like buffer size, time to live, message generation time, message size, and transmit speed are default.

\section{B. Simulation Results}

As described earlier that when vehicles are traveling in the same direction, their connection time will be long as compared to when they are crossing each other. Such scenarios are not considered where both are traveling in the same direction as the assumption is that in this case connection time will be always greater than the estimated time. Below there are simulation results and difference of time:

$$
\begin{aligned}
\mathrm{D} t & =\text { Difference time } \\
\mathrm{C} & =\text { Connection time } \\
\mathrm{E} \mathrm{t} & =\text { Estimated Time } \\
D t & =A b s(C t-E t)
\end{aligned}
$$

TABLE III. NO. OF ENCOUNTERS WITH DIFFERENT SPEEDS IN SECONDS

\begin{tabular}{|l|l|l|l|l|l|l|l|}
\hline $\begin{array}{l}\text { Sr. } \\
\text { No }\end{array}$ & $\begin{array}{l}\text { Vehicle/s } \\
\text { Speed/s }\end{array}$ & \multicolumn{6}{|l|}{ Time with Number of Vehicles } \\
\hline \multirow{2}{*}{} & \multicolumn{6}{|l|}{ Vehicles } \\
\cline { 3 - 8 } & 2 & 3 & 4 & 13 & 14 & 15 \\
\cline { 3 - 8 } & \multicolumn{2}{|l|}{ Encounters } \\
\hline 1 & $30-40$ & 62 & 151 & 324 & 4373 & 4936 & 5673 \\
\hline 2 & $41-50$ & 70 & 171 & 389 & 4922 & 5612 & 6310 \\
\hline 3 & $51-60$ & 79 & 180 & 415 & 5366 & 6098 & 6494 \\
\hline 4 & $61-70$ & 94 & 216 & 478 & 5574 & 6630 & 7512 \\
\hline
\end{tabular}

TABLE IV. Simulation Metrices and VALUes

\begin{tabular}{|l|l|l|}
\hline Sr. No & Metrices & Values \\
\hline 1 & Time & $43200 \mathrm{sec}$ \\
\hline 2 & Vehicle Speed & $30-70 \mathrm{KM} / \mathrm{h}$ \\
\hline 3 & No of Hosts & $2-15$ \\
\hline 4 & World Size & 4500,3400 \\
\hline 5 & Map & Helsinki City \\
\hline 6 & Transmit Range & 150 meters \\
\hline 7 & Wait Time & 0,120 \\
\hline
\end{tabular}


So, if the difference time is greater than 6.47 seconds as per reference to TABLE II, it is excluded from results with the assumption that it is Scenario A, Fig. 5, Fig. 6, Fig. 7. Fig. 8, Fig. 9 and Fig. 10 show the number of encounters and time difference. In x-axis, there is number of encounters of vehicles, that how many times any random two vehicles made a connection with each other. In y-axis, there is a time value, that for how long the connection was made. With increasing speed, number of "encounters" increases and connection "time" decreases.

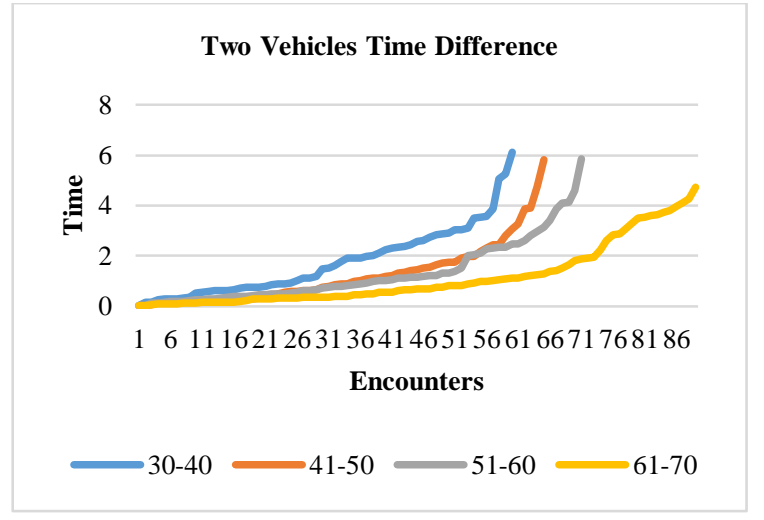

Fig. 5. Two Vehicles Time Difference.

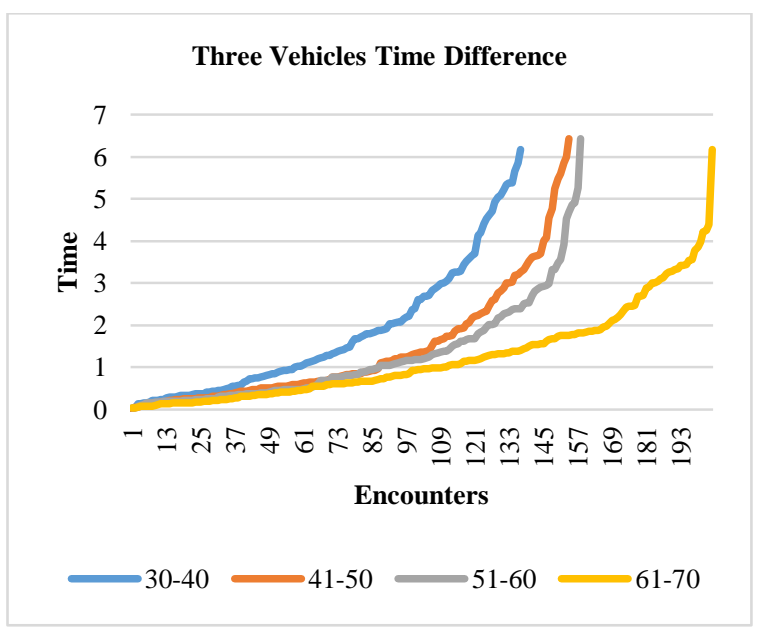

Fig. 6. Three Vehicles Time Difference.

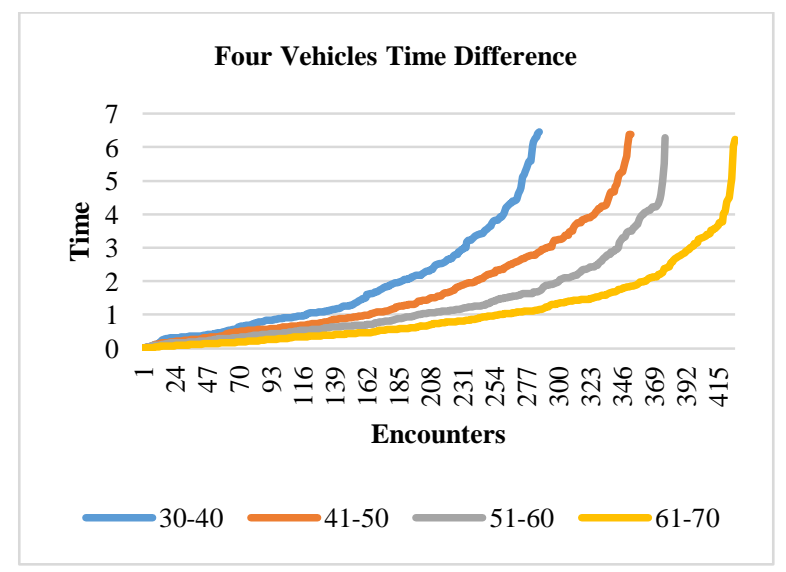

Fig. 7. Four Vehicles Time Difference.

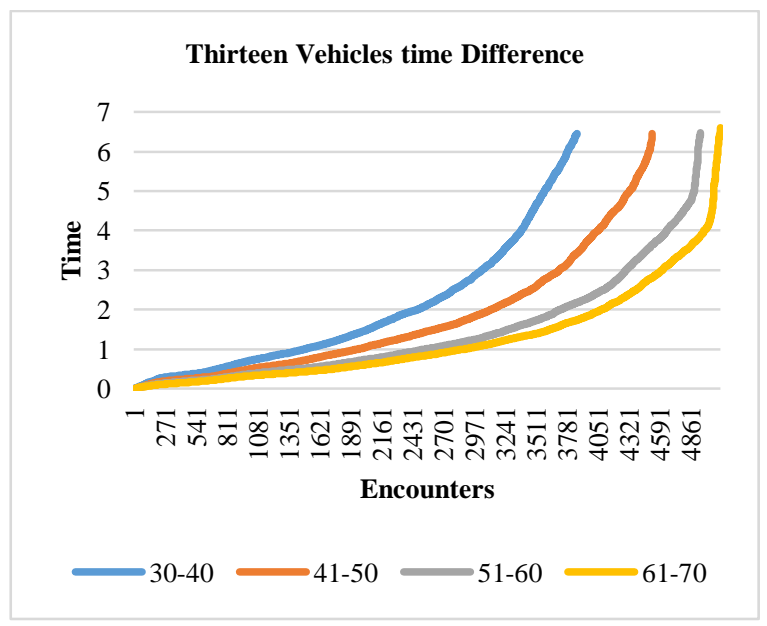

Fig. 8. Thirteen Vehicles Time Difference.

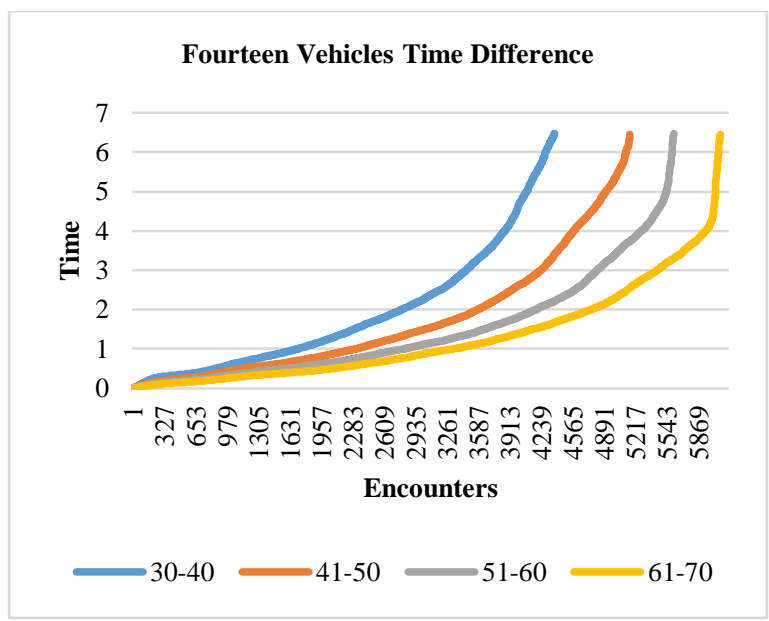

Fig. 9. Fourteen Vehicles Time Difference.

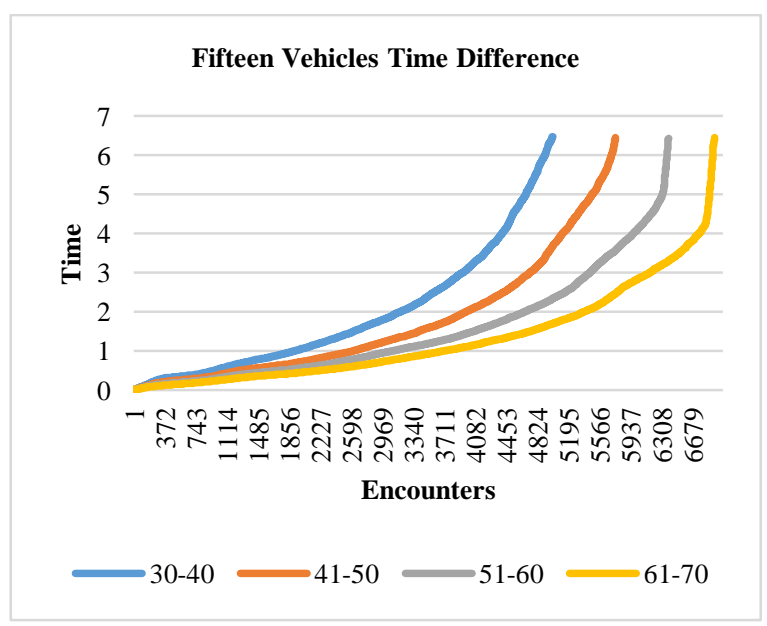

Fig. 10. Fifteen Vehicles Time Difference.

\section{Accuracy of Proposed estimated time:}

Difference time is equal to 4 seconds as base value. Below TABLE $V$ shows accuracy of estimation time.

Above table clearly shows that with increasing speed, accuracy is improved. Best results are with 61 to $70 \mathrm{KM} / \mathrm{H}$. 
TABLE V. ACCURACY OF ESTIMATED CONNECTION TIME IN SEONDS

\begin{tabular}{|l|l|l|l|l|l|l|}
\hline Speed & \multicolumn{6}{|l}{ Vehicles } \\
\hline & $\mathbf{2}$ & $\mathbf{3}$ & $\mathbf{4}$ & $\mathbf{1 3}$ & $\mathbf{1 4}$ & $\mathbf{1 5}$ \\
\hline $\mathbf{3 0 - 4 0}$ & 91.94 & 41.06 & 79.94 & 77.43 & 78.28 & 77.61 \\
\hline $\mathbf{4 1 - 5 0}$ & 90.00 & 84.21 & 83.80 & 82.36 & 81.59 & 81.25 \\
\hline $\mathbf{5 1 - 6 0}$ & 84.81 & 85.00 & 86.75 & 86.75 & 86.62 & 85.43 \\
\hline $\mathbf{6 1 - 7 0}$ & 91.49 & 92.59 & 88.37 & 89.38 & 89.67 & 89.96 \\
\hline
\end{tabular}

\section{CONCLUSION AND FUTURE WORK}

In vehicular delay tolerant networks routing decisions are taken based on multiple metrics like Time to Live (TTL), buffer size, buffer occupancy, geographic location and density of nodes, destination utility, relay utility, meeting prediction etc. This paper is about introducing a new decision metric called as the "estimation time". This metric can be used for routing decisions. Above $88 \%$ results show that it is possible to predict time at node's encounter. Multiple simulations were run in ONE simulator with different number of nodes to prove that "estimation time" work fine with different number of nodes. The "estimation time" can be used for routing decisions. In future, this metric will be used for routing decisions.

\section{REFERENCES}

[1] J. Yan and J. Liu, and F. M. Tseng, "An evaluation system based on the self-organizing system framework of smart cities: A case study of smart transportation systems in China," Technol. Forecast. Soc. Change, no. 1, pp. 1-12, 2018.

[2] N. Magaia et al., "Bundles fragmentation in Vehicular Delay-Tolerant Networks," in 2011 7th EURO-NGI Conference on Next Generation Internet Networks, NGI 2011 - Proceedings, pp. 1-6, 2011.

[3] S. N. Rana and M. S. Shah, and V. N. Pandya, "Performance Analysis and Improvement of VDTN with message copies," vol. 2, no. 3, pp. 136-141, 2014.

[4] C. Iwendi and M. Uddin and J. A. Ansere, P. Nkurunziza and J. H. Anajemba and A. K. Bashir, "On Detection of Sybil Attack in LargeScale VANETs Using Spider-Monkey Technique," IEEE Access, vol. 6, pp. 47258-47267, 2018.

[5] N. M. F. Qureshi et al., "An Aggregate MapReduce Data Block Placement Strategy for Wireless IoT Edge Nodes in Smart Grid," Wirel. Pers. Commun., pp. 1-12, 2018.

[6] A. Ali et al., "Priority-Based Cloud Computing Architecture for Multimedia-Enabled Heterogeneous Vehicular Users," J. Adv. Transp., vol. 2018, 2018.

[7] S. H. Chauhdary and A. Hassan and M. A. Alqarni, A. Alamri and A. K. Bashir, "A twofold sink-based data collection in wireless sensor network for sustainable cities," Sustain. Cities Soc., vol. 45, pp. 1-7, 2019.

[8] M. G. Rubinstein et al., "Measuring the capacity of in-car to in-car vehicular networks," IEEE Commun. Mag., vol. 47, no. 11, pp. 128136, Nov. 2009.

[9] V. N. G. J. Soares and J. J. P. C. Rodrigues and P. S. Ferreira and A. M. D. Nogueira, "Improvement of messages delivery time on vehicular delay-tolerant networks," in Proceedings of the International Conference on Parallel Processing Workshops, pp. 344-349, 2009.

[10] V. N. G. J. Soares and F. Farahmand and J. J. P. C. Rodrigues, "Improving vehicular delay-tolerant network performance with relay nodes," 2009 Next Gener. Internet Networks, NGI 2009, vol. 00, no. c, pp. 1-5, 2009.

[11] M. J. Khabbaz and W. F. Fawaz,and C. M. Assi, "A probabilistic bundle relay strategy in two-hop vehicular delay tolerant networks," IEEE Int. Conf. Commun., vol. 15, no. 3, pp. 281-283, 2011.
[12] D. Wu and Q. Yang and D. Zhao, "Adaptive Carry-Store Forward Scheme in Two-Hop Vehicular Delay Tolerant Networks," IEEE Commun. Lett., vol. 17, pp. 721-724, 2013.

[13] W.-Z. Lo and J.-S. Gao and S.-C. Lo, "Distance-Aware Routing with Copy Control in Vehicle-Based DTNs," 2012 IEEE 75th Veh. Technol. Conf. (VTC Spring), pp. 1-5, 2012.

[14] Q. Fu and B. Krishnamachari and L. Zhang, "DAWN: A density adaptive routing for deadline-based data collection in vehicular delay tolerant networks," Tsinghua Sci. Technol., vol. 18, no. 3, pp. 230-241, 2013.

[15] I. Leontiadis and C. Mascolo, "GeOpps: Geographical Opportunistic Routing for Vehicular Networks," in 2007 IEEE International Symposium on a World of Wireless, Mobile and Multimedia Networks, pp. 1-6, 2007.

[16] V. N. G. J. Soares and J. J. P. C. Rodrigues and F. Farahmand, "GeoSpray: A geographic routing protocol for vehicular delay-tolerant networks," Inf. Fusion, vol. 15, no. 1, pp. 102-113, 2014.

[17] S. Jain and K. Fall and R. Patra, "Routing in a Delay Tolerant Network," SIGCOMM Comput. Commun. Rev., vol. 34, no. 4, pp. 145-158, Aug. 2004.

[18] T. Spyropoulos and K. Psounis and C. S. Raghavendra, "Single-copy routing in intermittently connected mobile networks," in 2004 First Annual IEEE Communications Society Conference on Sensor and Ad Hoc Communications and Networks, 2004. IEEE SECON, pp. 235-244, 2004.

[19] A. Vahdat and D. Becker, "Epidemic routing for partially connected ad hoc networks," Tech. Rep. number CS-200006, Duke Univ., no. CS200006, pp. 1-14, 2000.

[20] T. Spyropoulos and K. Psounis and C. S. Raghavendra, "Spray and Wait: An Efficient Routing Scheme for Intermittently Connected Mobile Networks," in Proceedings of the 2005 ACM SIGCOMM Workshop on Delay-tolerant Networking, pp. 252-259, 2005.

[21] A. Lindgren and A. Doria and O. Schelén, "Probabilistic Routing in Intermittently Connected Networks," SIGMOBILE Mob. Comput. Commun. Rev., vol. 7, no. 3, pp. 19-20, Jul. 2003.

[22] R. Ramanathan and P. Basu and R. Krishnan, "Towards a formalism for routing in challenged networks," in Proceedings of the second workshop on Challenged networks CHANTS - CHANTS '07, p. 3, 2007.

[23] M. Cuka and I. Shinko and E. Spaho and T. Oda and M. Ikeda and L. Barolli, "A simulation system based on ONE and SUMO simulators: Performance evaluation of different vehicular DTN routing protocols," J. High Speed Networks, vol. 23, no. 1, pp. 59-66, 2017.

[24] A. Mehto and M. Chawla, "Comparing Delay Tolerant Network Routing Protocols for Optimizing L-Copies in Spray and Wait Routing for Minimum Delay," Int. Conf. Adv. Commun. Control Syst., vol. 2013, no. Cac2s, pp. 239-244, 2013.

[25] A. H. Cherif and K. Boussetta and G. Diaz and D. Fedoua, "Improving the performances of geographic VDTN routing protocols," 2017 16th Annu. Mediterr. Ad Hoc Netw. Work. Med-Hoc-Net 2017, no. 1, pp. 14, 2017.

[26] A. S. S. Vieira and J. G. Filho and J. Celestino and A. Patel, "VDTNToD: Routing protocol VANET/DTN based on trend of delivery," Adv. Int. Conf. Telecommun. AICT, vol. 2013-Janua, no. January, pp. 135141, 2013.

[27] M. R. Penurkar and U. A. Deshpande, "Max-Util: A Utility-Based Routing Algorithm for a Vehicular Delay Tolerant Network Using Historical Information," in Proceedings of 3rd International Conference on Advanced Computing, Networking and Informatics, pp. 587-598, 2016.

[28] E. M. Salvador and D. F. Macedo and J. M. Nogueira and V. Del Duca Almeida and L. Z. Granville, "Hierarchy-based monitoring of Vehicular Delay-Tolerant Networks," 2016 13th IEEE Annu. Consum. Commun. Netw. Conf. CCNC 2016, pp. 447-452, 2016.

[29] M. R. Penurkar and U. A. Deshpande, "Priority-based scheduling policy for a hybrid routing algorithm in a Vehicular Delay Toleant Network," in 2016 International Conference on Computing, Analytics and Security Trends (CAST), pp. 578-583, 2016. 
[30] K. Bylykbashi and E. Spaho and L. Barolli and F. Xhafa, "Routing in a many-to-one communication scenario in a realistic VDTN," J. High Speed Networks, vol. 24, no. 2, pp. 107-118, 2018.

[31] N. P. M. Magaia and C. Borrego and P. Pereira and M. P. Correia, "ePRIVO: An enhanced PRIvacy-preserVing Opportunistic routing protocol for Vehicular Delay-Tolerant Networks," IEEE Trans. Veh. Technol., vol. 9545, no. c, pp. 11154-11168, 2018.

[32] H. Rafique and F. Anwer and A. Shamim and B. Minaei-Bidgoli and M. A. Qureshi and S. Shamshirband, "Factors Affecting Acceptance of Mobile Library Applications: Structural Equation Model,” Libri, vol. 68, no. 2, pp. 99-112, 2018.
[33] Adnan Ali, Nadeem Sarwar, Hamaad Rafique, Imtiaz Hussain, Faheem Nawaz Khan, "Connection Time for Routing Decisions in Vehicular Delay Tolerant Network" in press

[34] A. Keränen, J. Ott and T. Kärkkäinen, "The ONE Simulator for DTN Protocol Evaluation," in SIMUTools '09: Proceedings of the 2nd International Conference on Simulation Tools and Techniques, 2009. 